\title{
Philosophiques
}

\section{La prudence de Descartes face à la question de l'infini en mathématiques}

\section{Jean-Baptiste Jeangène Vilmer}

Volume 34, numéro 2, automne 2007

URI : https://id.erudit.org/iderudit/016991ar

DOI : https://doi.org/10.7202/016991ar

Aller au sommaire du numéro

Éditeur(s)

Société de philosophie du Québec

ISSN

0316-2923 (imprimé)

1492-1391 (numérique)

Découvrir la revue

\section{Citer cet article}

Jeangène Vilmer, J.-B. (2007). La prudence de Descartes face à la question de l'infini en mathématiques. Philosophiques, 34(2), 295-316.

https://doi.org/10.7202/016991ar

\section{Résumé de l'article}

La question de l'infini cartésien est vaste et polymorphe, de la métaphysique à la philosophie des sciences en passant par la philosophie pratique. Mais c'est en mathématiques que l'attitude de l'auteur est la plus ambivalente et paradoxale, car il n'y a pas, chez Descartes, d'infini en mathématiques. Le but de cet article est d'analyser les manifestations et les raisons de cette prudence cartésienne. Pour ce faire, nous procédons en deux temps. D’abord, nous constatons l'absence d'infini en mathématiques à travers l'examen de l'infinitésimal. Ensuite, nous montrons qu'il n'y a, dans les mathématiques cartésiennes, qu'un indéfini, en discutant l'existence et le statut du " plus grand nombre ». Ce parcours dévoilera les deux motifs de la prudence cartésienne : la contrainte métaphysique et la rigueur méthodologique. 


\title{
La prudence de Descartes face à la question de l'infini en mathématiques
}

\author{
JEAN-BAPTISTE JEANGÈNE VILMER \\ Université de Montréal / McGill University / EHESS \\ jb.jeangene.vilmer@umontreal.ca
}

\begin{abstract}
RÉSUMÉ. — La question de l'infini cartésien est vaste et polymorphe, de la métaphysique à la philosophie des sciences en passant par la philosophie pratique. Mais c'est en mathématiques que l'attitude de l'auteur est la plus ambivalente et paradoxale, car il n'y a pas, chez Descartes, d'infini en mathématiques. Le but de cet article est d'analyser les manifestations et les raisons de cette prudence cartésienne. Pour ce faire, nous procédons en deux temps. D'abord, nous constatons l'absence d'infini en mathématiques à travers l'examen de l'infinitésimal. Ensuite, nous montrons qu'il n'y a, dans les mathématiques cartésiennes, qu'un indéfini, en discutant l'existence et le statut du «plus grand nombre ». Ce parcours dévoilera les deux motifs de la prudence cartésienne : la contrainte métaphysique et la rigueur méthodologique.
\end{abstract}

Mots-clés: Descartes, mathématiques, infini, indéfini, infinitésimal, nombre.

\begin{abstract}
The question of the Cartesian infinite is vast and polymorphic, from metaphysics to the philosophy of science and practical philosophy. But it is in mathematics that the attitude of the author is the most ambivalent and paradoxal because, for Descartes, there is no infinite in mathematics. This article aims to analyze the signs and the reasons of this cartesian prudence. We will proceed in two steps. Firstly, we notice the absence of the infinite in Cartesian mathematics through the examination of the infinitesimal. Secondly, we will demonstrate that there is, in Cartesian mathematics, only an indefinite, by discussing the existence and the status of the «biggest number». This examination will reveal the two motives of cartesian prudence : metaphysical constraint and methodological rigour.
\end{abstract}

Keywords: Descartes, mathematics, infinite, indefinite, infinitesimal, number.

L'infini est une grille de lecture privilégiée de l'œuvre cartésienne. De la métaphysique à la philosophie pratique en passant par les sciences, la distinction que Descartes opère entre infini (infinitus) et indéfini (indefinitus) traverse et éclaire les textes, de Dieu au monde, de l'univers à la volonté, de l'atome au plus grand nombre. Une difficulté, cependant, persiste : l'infini en mathématiques. Quand on connaît à la fois l'importance et l'ambivalence du rôle des mathématiques dans l'environnement cartésien, l'attitude de l'auteur, souvent sévère, toujours critique, envers les problèmes posés par ses contemporains, on ne s'étonnera pas que l'infini y occupe une place à l'arrière-scène. La position de Descartes face à la question de l'infini en mathématiques est complexe, 
paradoxale, prudente. Pour le dire en un mot: il n'y a pas, chez Descartes, d'infini en mathématiques.

À plusieurs endroits, l'auteur fait de sévères critiques à l'encontre des mathématiques, évoquant «les problèmes creux avec lesquels les arithméticiens ou les géomètres ont coutume d'amuser leurs loisirs ${ }^{1}$ » : « Rien n'est plus vain que de s'occuper de nombres abstraits et de figures imaginaires, au point de sembler vouloir se contenter de connaître de pareilles bagatelles ${ }^{2}$.» En 1630, il écrivait déjà à Mersenne: «Pour des problèmes, je vous en enverrai un million pour proposer aux autres, si vous le désirez; mais je suis si las des mathématiques, et en fais maintenant si peu d'état, que je ne saurais plus prendre la peine de les soudre moi-même ${ }^{3}$.»

L'attitude de Descartes, loin d'être un pur rejet, dissimule en vérité un simple reproche: si l'auteur ne s'occupe guère des mathématiques et en fait un seul loisir, c'est en remarquant combien sont vains les problèmes qu'elle pose, c'est-à-dire combien son abstraction manque l'essentielle visée pratique qui doit être celle de l'esprit humain. Les mathématiques cartésiennes, car elles sont malgré tout, ont effectivement, elles, une visée résolument pratique et ordinaire ${ }^{4}$. Et s'il en est ainsi, c'est que les mathématiques, pour Descartes, ne sont rien de plus qu'une fabrication de l'esprit — thèse qui domina l'école française de la fin du XIX ${ }^{\mathrm{e}}$ siècle et du début du XX $\mathrm{XX}^{\mathrm{e}}$ (Borel, Poincaré, Lebesgue), dont on retrouve encore aujourd'hui les traces dans le constructivisme, et qui oppose ses mathématiques du faire à celles de l'être, défendues notamment par Cantor et Dedekind.

Dans un tel contexte, on comprendra aisément que la question de l'infini, que déjà Descartes se refuse à expliciter d'une manière générale, en raison de son essentielle incompréhensibilité, soit, en mathématiques, plus encore qu'ailleurs, évitée, dans la lignée de la tradition grecque ${ }^{5}$. L'auteur fait la promesse de ne rien déterminer de cette question:

Ainsi nous ne nous embarrasserons jamais dans les disputes de l'infini; d'autant qu'il serait ridicule que nous, qui sommes finis, entreprissions d'en déterminer quelque chose, et par ce moyen le supposer fini en tâchant de le comprendre; c'est pourquoi nous ne nous soucierons pas de répondre à ceux qui demandent si la moitié d'une ligne infinie est infinie, et si le nombre infini est pair ou non pair, et d'autres choses semblables, à cause qu'il n'y a que ceux qui s'imaginent que leur esprit est infini qui semblent devoir examiner telles difficultés ${ }^{6}$.

C'est précisément parce que Descartes n'entend rien déterminer de la question de l'infini en mathématiques, que nous pourrons assurer, d'une part, qu'il

1. Regulae IV; AT X 373.

2. Regulae IV; AT X 375.

3. À Mersenne du 15 avril 1630; AT I 139.

4. Voir Regulae IV; AT X 373-374.

5. Comme le souligne Belaval, 1960, p. 302.

6. Principes I, 26; AT IX-2 36. 
n'y a pas, chez Descartes, d'infini mathématique et, d'autre part, que les rares réponses que l'auteur ne parvient pas à taire touchant cette question ne laissent voir que son refus d'en déterminer quoi que ce soit, c'est-à-dire l'in-détermination de l'in-défini'.

En premier lieu, s'il n'y a pas, chez Descartes, d'infini mathématique, c'est que l'infini cartésien — l'infinita substantia — exclut que l'on puisse parler d'un autre que lui, qui serait un infini non véritable, et qui pourrait œuvrer en mathématiques. Leibniz ne sera pas si exigeant et proposera que l'on puisse disputer de l'infini en mathématiques, à condition de savoir que cet infini n'est pas le véritable: à Des Bosses, qui lui oppose, d'une manière éminemment cartésienne, «4- Notre esprit, de ce qu'il est fini, ne peut rien savoir de certain sur l'infini, et partant il convient de n'en jamais disputer ", il répond:

Les mathématiciens, si je ne m'abuse, ont déjà réfuté la quatrième, et j'ai moimême donné quelques spécimens de la science de l'infini. En attendant, je pense qu'à proprement parler l'infini formé de parties n'est ni une unité ni un tout, et qu'on ne le conçoit comme quantité que par pure fiction de l'esprit. Seul l'infini sans parties est un, mais il n'est pas un tout; cet infini est Dieu'.

Mais Descartes, quant à lui, interdit que l'infinita substantia puisse côtoyer, et être comme limité par lui, un infini en son genre qui ne serait pas l'infini en tous les genres. La notion d' "infini mathématique » est absurde, dans la mesure, comme le rappellera le cartésianisme de Fénélon, où «l'infini ne peut admettre ni nombre ni augmentation ${ }^{9} »$. Et c'est pourquoi il n'y a pas, à proprement parler, d'infini en mathématiques.

Et en second lieu, si Descartes reste donc indécis sur la question de l'infini en mathématiques, c'est aussi, tout simplement, parce que la mathématique « accoutume à se repaître de vérités (à reconnaître la vérité) ${ }^{10}$ » et que, sur des problèmes tels que ceux qui demandent «si la moitié d'une ligne infinie est infinie, et si le nombre infini est pair ou non pair, et d'autres choses semblables ${ }^{11}$ », on ne saurait prétendre dire une vérité, puisque la vérité, dans ce cas, est au-delà des capacités de notre esprit fini. Or, en ne faisant de la question de l'infini en mathématique que celle de l'indéfini, Descartes s'autorise, non à dire une quelconque vérité sur ces problèmes, mais seulement à décrire l'impuissance dans laquelle il est de ne pouvoir le faire.

Nous bâtirons donc la thèse selon laquelle, chez Descartes, étudier la question de l'infini en mathématiques ne revient qu'à dévoiler et expliquer les signes de sa fuite, en deux moments : d'abord, nous verrons comment il n'y a pas, chez Descartes, d'infini mathématique par le biais de la position

7. Sur ce point, voir notre article «La véritable nature de l'indéfini cartésien ", à paraître.

8. Leibniz, 1960, t. II, p. 313.

9. Fénélon, 1712, $2^{\mathrm{e}}$ partie, chap. V, art. $1, \mathbb{\text { II. }}$

10. Entretien avec Burman; AT V 177; Descartes, 1981, p. 142.

11. Principes I, 26; AT IX-2 36. 
cartésienne touchant la question de l'infinitésimal. Ensuite, nous conclurons qu'il n'y a, chez Descartes, et sur l'infini en mathématiques, qu'un indéfini, en discutant l'existence et le statut du «plus grand nombre» .

\section{L'infinitésimal}

Si l'on pense presque systématiquement à Leibniz, en ce qui concerne la question de l'invention du calcul infinitésimal, on oublie trop souvent trois sources. Premièrement, le travail initiateur de Fermat qui, dès 1632, dans son De Maximis et Minimis, utilise ce qui pourrait être tenu pour le premier calcul infinitésimal, à savoir la première méthode d'algébrisation permettant de trouver la tangente à une courbe: dans ses calculs, Fermat use effectivement d'une quantité infinitésimale, notée $e$, et qui, bien que non nulle, peut être supprimée; ainsi passe-t-il de l'adégalité « $\mathrm{b}(2 \mathrm{a}+e$ » à l'égalité « $\mathrm{b}=2 \mathrm{a}$ ». Reste que l'infinitésimal n'est pas chez Fermat la plus belle manifestation de la question de l'infini en mathématiques: il s'agirait plutôt de la descente infinie (ou indéfinie: Fermat sur ce point utilise la même précaution de vocabulaire), laquelle peut être considérée comme le point de départ du courant constructiviste sur la question, comme on peut le voir chez Yvon Gauthier. Deuxièmement, Pascal, dont Leibniz lui-même reconnaîtra l'influence sur son propre calcul ${ }^{12}$, et qui écrit à Fermat, comme à son allié, au sujet de son adversaire le Chevalier de Méré:

Je n'ai pas le temps de vous envoyer la démonstration d'une difficulté qui étonnait fort M. de Méré, car il a très bon esprit, mais il n'est pas géomètre; c'est, comme vous savez, un très grand défaut, et même il ne comprend pas qu'une ligne mathématique soit divisible à l'infini et croit fort bien entendre qu'elle est composée de points en nombre fini et jamais je n'ai pu l'en tirer; si vous pouviez le faire, on le rendrait parfait ${ }^{13}$.

Et troisièmement, Newton, dont la méthode des fluxions n'est pas non plus à négliger ${ }^{14}$.

Et s'il est si répandu de faire naître le calcul infinitésimal avec Leibniz, c'est qu'il est tout autant remarquable que Descartes l'a à ce titre effleuré, ne pouvant revendiquer pour sa part que la paternité de la géométrie moderne,

12. Faisant référence à une lettre de M. Dettonville de la fin de 1658 (Amos Dettonville est le pseudonyme que Pascal prend fin 1658) à M. de Carcavi et, parlant de lui-même, Leibniz écrit, dans son Histoire et origine du Calcul différentiel: «De retour d'Angleterre en France vers 1673, l'auteur se mit, sur le conseil de Huygens, à étudier l'Analyse cartésienne, et pour recevoir une introduction à la géométrie des quadratures, il consulta : la Vue d'ensemble de la géométrie de l'estimable Fabri, Grégoire de Saint-Vincent et un petit livre de Dettonville (c'est-à-dire de Pascal) ». Le texte de Pascal en question est celui de la Lettre de A. Dettonville à Monsieur de Carcavy, imprimée entre décembre 1658 et janvier 1659, et reproduite dans Pascal, 1908-1914, t. VIII, p. 325-384.

13. Pascal à Fermat du 29 juillet 1654, dans Pascal, 1908-1914, t. III, p. 375-393.

14. Cléro, 1989-90, et Gandt, 1992, soulignent combien, dans l'invention du calcul infinitésimal, Newton a joué un rôle aussi important que celui de Leibniz. 
c'est-à-dire algébrique (ou analytique ${ }^{15}$ ), comme le rappelle Louis Couturat, répartissant ainsi les mérites: «Descartes, le père de la Géométrie moderne, et Leibniz, l'inventeur du Calcul infinitésimal ${ }^{16}{ }^{\prime}$. Il s'agit donc pour nous de comprendre dans quelle mesure on peut reconnaître à Descartes pour une petite part d'en avoir été le précurseur, et pour une grande part de l'avoir formidablement - et délibérément - manqué.

\section{A. La réticence de Descartes face à la question de l'infinitésimal}

En quoi Descartes peut-il être vu comme un précurseur du calcul infinitésimal, voilà qui peut être réglé rapidement, car si l'on doit reconnaître qu'il s'approche parfois très près des prémisses de l'infinitésimal, le fait qu'il feigne de ne point le remarquer pour l'éviter soigneusement ampute systématiquement les ambitions qu'on lui pourrait attribuer. Certains interprètes, comme Sergescu, persistent malgré tout à lui accorder, sur ce point, un rôle non négligeable: «Il faut dire que Descartes (1596-1650), sans s'occuper de la notion d'infini mathématique, a joué un très grand rôle dans le développement de tous ces calculs infinitésimaux; on verra plus loin que sans lui on n'aurait pas pu avoir Leibniz ${ }^{17}$. » Pour quelles raisons, donc, peut-on reconnaître que Descartes, d'une certaine manière, a conditionné le calcul infinitésimal à venir ?

On pourrait voir au moins deux arguments. D'une part, dans la mesure où le calcul différentiel, qui exige des méthodes infinitésimales, est lui-même construit sur la base de l'algèbre cartésienne, on peut indirectement accorder à Descartes, certes à son corps défendant, un rôle dans la genèse du calcul infinitésimal, même sans qu'il ait jamais lui-même joint l'usage des séries infinies à son algèbre pour engendrer ce qui aurait pu devenir le calcul différentiel ${ }^{18}$. Et, d'autre part, on peut aussi attribuer à Descartes la découverte d'un principe essentiel du calcul infinitésimal, pour avoir été le premier à voir que l'intégration est une opération inverse de la détermination de la tangente à une courbe (c'est-à-dire la différenciation), dans une lettre à Florimond de Beaune ${ }^{19}$.

Néanmoins, et quant à se demander si Descartes, pour ces deux raisons, possédait en conséquence une sorte d'équivalent du calcul infinitésimal avant l'heure, Vuillemin prévient fort justement que

l'on a coutume, depuis, Lazare Carnot ${ }^{20}$, de prétendre que Descartes possédait un équivalent du Calcul infinitésimal dans la méthode des indéterminées. Cette

15. Bien qu'étant la plupart du temps dite " analytique ", sa véritable nature est sans conteste algébrique : l'analytique ne touchant que sa lecture. Voir Brunschvicg, 1904, p. 770 : «La géométrie de Descartes [...] est proprement une algèbre »; et 1927, p. 286 : «Il crée une géométrie qui est avant tout une algèbre. »

16. Couturat, 1896, p. 265.

17. Sergescu, 1955 , p. 70.

18. C'est la conclusion de Houzel, 1997, p. 35.

19. À Florimond de Beaune du 20 février 1639; AT II, 513-514.

20. Voir Carnot, 1797 et, sur lui, Gillispie et Iushkevich, 1979. 
affirmation n'est véritable qu'à une double condition : d'abord faire abstraction du fondement que Carnot prête à cette méthode, y voyant faussement à l'œuvre une compensation d'erreurs; ensuite en éliminer toute notion d' "équation imparfaite " et d'approximation, étrangères à l'Algèbre ${ }^{21}$.

Et il se trouve que Descartes, précisément, se rend aveugle de la naissance du calcul infinitésimal pour y refuser l'approximation, selon les exigences de son algèbre propre. C'est ainsi qu'il y a bien plus à dire, concernant la question de l'infinitésimal chez Descartes, en examinant la manière dont il a su, non y répondre, ou même la poser, mais tout simplement la contourner. On retiendra trois expressions.

Premièrement, par la seule attitude de Descartes, qui reproche à ses correspondants de travailler l'infinitésimal, ou encore leur dénonce les travaux de ceux qui s'y occupent. L'attitude générale de Descartes sur la question des infiniment petits en mathématiques est effectivement, et sans conteste, la plus simple réticence, comme en témoigne sa correspondance avec Fermat et sur Galilée.

La querelle qui oppose Descartes à Fermat ${ }^{22}$ révèle précisément en quoi la critique cartésienne de la conception de la tangente comme d'un maximum ou minimum d'un segment sécant à la parabole est celle de l'infinitésimal et, plus largement, de la question de l'infini en mathématiques. Ce que Descartes refuse à Fermat est l'évanouissement d'une quantité quelconque : en passant de l'adégalisation qui approxime $e$ comme tendant vers 0 à l'égalité qui l'y rend identique pour pouvoir la rendre évanescente, Fermat semble considérer une même quantité à la fois comme différente de 0 et comme nulle, ce qui implique une contradiction, et est problématique. Un siècle plus tard, Berkeley formulera la même critique des méthodes infinitésimales en doutant notamment de la validité d'une mathématique qui utiliserait une grandeur non nulle mais tenue pour nulle. Dans un court texte intitulé, Of Infinites, et présenté le 19 novembre 1707, Berkeley montre qu'un infinitésimal "n'est absolument rien ${ }^{23} »$. En 1734, dans The Analyst, il développera méthodiquement de nombreuses objections à l'encontre des méthodes infinitésimales de toutes sortes.

Descartes, en un mot, ne se permet pas ce saut de l'infiniment petit au néant, puisqu'entre les deux il ne saurait y avoir une différence de degré c'est précisément la raison d'être de l'infinitésimal, qui reste hors du 0 pour ne jamais épuiser tous les degrés qui, théoriquement, mènent à lui — mais de nature. C'est pourquoi la correspondance de Descartes avec Fermat montre que le premier pense du second qu'il est bon mathématicien (on ajoutera : sans doute, même, supérieur à Descartes), mais piètre philosophe.

21. Vuillemin, 1960, p. 62.

22. Sur la correspondance Descartes - Fermat, voir Mahoney, 1973, p. 170-193.

23. Berkeley, 1985, p. 146. 
Quant à Galilée $e^{24}$, Descartes écrit à Mersenne que son procédé annonçant le calcul infinitésimal lui semble n'être qu'un sophisme:

[Page $19^{25}$ ] Il examine la colle qu'il ajoute avec le vide pour la liaison des parties des corps, et il l'attribue à d'autres petits vides qui ne sont nullement imaginables. Et ce qu'il dit (p. 22), pour prouver ces petits vides, est un sophisme; car l'hexagone qu'il propose ne laisse rien de vide en l'espace par où il passe, mais chacune de ses parties se meut d'un mouvement continu, lequel décrivant des lignes courbes qui remplissent tout un espace, on ne doit pas les considérer, comme il fait, en une seule ligne droite. Et il n'importe qu'en sa figure les parties de la ligne droite, IO, PY, etc., ne soient point touchées par la circonférence H I K L, car elles le sont en récompense par d'autres parties de la superficie A B C, et ainsi ne sont non plus vides que les parties OP, YZ, etc. [Page 28] C'est aussi un sophisme que son argument, pour prouver qu'un point est égal à une ligne ou à une superficie. Car in forma on ne peut conclure, sinon que la ligne ou superficie n'est pas un plus grand corps solide que le point, et non qu'elle n'est pas plus grande absolument. [Page 31] Il manque en tout ce qu'il dit de l'infini, en ce que, nonobstant qu'il confesse que l'esprit humain, étant fini, n'est pas capable de le comprendre, il ne laisse pas d'en discourir tout de même que s'il le comprenait ${ }^{26}$.

On notera que Galilée lui-même reconnaît que notre entendement fini ne peut comprendre adéquatement ces problèmes touchant l'infini, mais, contrairement à Descartes, qui peut-être est plus exigeant, il invoque notre aspiration vers cet infini pour défendre le fait d'y travailler malgré tout : "Rappelonsnous que nous traitons d'infinis et d'indivisibles, inaccessibles à notre entendement fini, les premiers à cause de leur immensité, les seconds à cause de leur petitesse. Pourtant nous constatons que la raison humaine ne peut s'empêcher de sans cesse y revenir ${ }^{27}$."

Deuxièmement, l'esquive cartésienne se manifeste également dans le problème de la quadrature de la cycloïde, pour lequel Descartes ne s'embarrasse pas de l'infini. On lit dans les lettres à Mersenne des 27 mai et 27 juillet 1638 que, pour conclure à l'égalité de deux surfaces (l'arc de la cycloïde et un cercle appelé générateur), Descartes ne conçoit pas chacune des surfaces comme une somme (infinie) de lignes droites ${ }^{28}$, ce qui exigerait de créer un algorithme d'intégration,

24. Sur Descartes et Galilée, voir Enriques, 1937; Banfi, 1957; et Marion, 1981, p. 203227. Plus précisément, sur la critique que fait Descartes de Galilée, voir Shea, 1978.

25. Descartes commente le livre de Galilée Discorsi e dimostrazioni matematiche, intorno a due nuove scienze (Discours concernant deux sciences nouvelles) récemment paru le 6 mars 1638 .

26. À Mersenne du 11 octobre 1638; AT II, 382-384. Alquié, 1992, p. 94, n. 2, objecte que Descartes agit exactement de même: «cette remarque est curieuse, Descartes agissant exactement de la même façon ». Il faut lui répondre que, si c'était vraiment le cas, nous aurions peut-être une doctrine systématisée de l'infinité chez Descartes. Mais, au contraire, il semble que Descartes n'en discute pas beaucoup. Et pour ce qu'il en discute, il le fait tout en signalant constamment l'incompréhensibilité de l'infini, ce qui n'est pas négligeable.

27. Galilée, 1970, p. 26.

28. Notamment en raison de l'infinie divisibilité de la matière: on ne cessera jamais de trouver une droite plus étroite que la précédente. 
mais il se contente de faire passer par n'importe quel point de leur hauteur une parallèle qui sera égale dans l'une et dans l'autre des figures (de telle sorte qu'il pourra conclure à leur égalité). Et, sur ce point, il est si proche de Cavalieri ${ }^{29}$ - l'«inventeur » d'une telle méthode — qui résout le problème en balayant de la même façon et par une règle les deux surfaces, qu'on pourrait lui reconnaître, comme le fait Belaval, de l'avoir même mieux compris que ne le fera Leibniz ${ }^{30}$. Pascal, à l'inverse, s'insurgera que l'on ne veuille point donner de sens à l'expression "somme de droites", et sera en conséquence bien moins prudent de s'embarrasser des disputes de l'infini, n'hésitant pas à parler de la "somme de tous les arcs " d'un cercle ou encore d'une "somme d'une multitude indéfinie de lignes ", alors même que les définitions de telles expressions restent floues ${ }^{31}$.

Troisièmement, il faut enfin examiner l'exclusion des passages à la limite $^{32}$. C'est certainement là l'exemple le plus manifeste de la manière dont Descartes a soigneusement évité l'infinitésimal et tout ce qui était présupposé par lui ou pouvait y conduire. On le remarque à plusieurs endroits.

En métaphysique, tout d'abord, parce que le passage à la limite, qui ici est celui d'une quantité infinitésimale au néant du 0 , serait celui du fini à l'infini, alors même que la première règle, touchant l'infini, est celle du tout ou rien, qui interdit qu'une échelle de degrés, même de longueur infinie, puisse le joindre au fini et le décliner en une infinité d'infinis dégradés ${ }^{33}$.

En physique, également, à travers la discontinuité entre repos et mouvement et la négation que la vitesse doit passer par tous les degrés pour le mouvement accéléré ${ }^{34}$, sans compter la solution à l'« Achille » de Zénon qui, en montrant comment la somme des termes d'une série convergente ne tend pas

29. Ce même Cavalieri qui, huit ans plus tard, et toujours dans une lettre à Mersenne, se verra, cette fois, et selon l'auteur, redevable de Descartes: "Ce Cavalieri ne fait autre chose que de démontrer, par un nouveau moyen, des choses qui ont déjà été démontrées par d'autres. » Descartes reconnaît alors: «Ce nouveau moyen n'est autre que l'un de ceux dont je me suis servi pour démontrer la roulette, en supposant que deux triangles curvilignes différents étaient égaux, pour ce que toutes les lignes droites tirées en même sens en l'un qu'en l'autre étaient égales " (à Mersenne le 20 avril 1646; AT IV, 395).

30. Belaval, 1960, p. 302.

31. En cela, ne déroge-t-il pas à sa propre règle qui demande de "n'employer dans la définition des termes que des mots parfaitement connus ou déjà expliqués »? (Pascal, 1908-1914, t. IX, p. 279.)

32. Le passage à la limite est, par exemple, l'équation suivante : $1 / 2+1 / 4+1 / 8+\ldots+1 / 2 n$ $+\ldots=1$. Lorsque l'on demande alors : quel est le sens de l'égalité ? on devrait répondre : a priori, elle a bien peu de sens, puisque la sommation ne peut être achevée. C'est pourquoi, après Cauchy, on écrira : limite de cette somme $=1$.

33. On prendra "dégradés » dans les deux acceptions : en dégradant l'infini, c'est-à-dire en le diluant, par degrés, dans le non infini, on le dégrade, du point de vue de sa valeur : il perd sa perfection, et du même coup son infinité.

34. Descartes, dans les lettres à Mersenne des 17 novembre et 7 décembre 1642, réfute l'idée qu'un corps qui se met en mouvement doive passer par tous les degrés de vitesse, en donnant l'exemple d'un grand corps dur qui percute dans son mouvement un petit corps au repos: ce dernier prend alors immédiatement sa vitesse finale. 
vers une limite finie, mais est égale à une quantité finie, s'extrait de toute compromission avec l'idée de limite.

Il ne faut pas croire ensuite que la courbe proposée à de Beaune, évoquée tout à l'heure, et qui semble utiliser la méthode des limites, le fasse réellement, puisque, comme le rappelle Yvon Belaval, «telle qu'elle se présente chez lui, elle procède par exhaustion de "parties» finitésimales — et non infinitésimales; elle reste étrangère à la notion de convergence qui ne sera dégagée que par Gregory et reprise par l'anticartésien Wallis ${ }^{35}$ ».

Enfin, le refus d'utiliser la notion de " passage à la limite » doit finalement se comprendre à partir de l'idée de limite elle-même, que Descartes ne se résigne pas à accepter claire et distincte, comme le note Vuillemin :

L'idée de limite, dont le philosophe sent au moins confusément qu'elle fonde le Calcul infinitésimal, et qui implique celle d'approximation à l'infini, ne saurait être reçue par lui comme exacte ou distincte. Les courbes transcendantes assignent donc à l'entendement tel qu'il le définit en Mathématiques une limite infranchissable, qui leur confère dans le système de Descartes un rôle analogue à celui que jouent chez Kant les idées pures de la raison que nous ne saurions nous représenter dans une intuition sensible ${ }^{36}$.

C'est d'ailleurs pourquoi Belaval reproche justement à Gueroult d'avoir fait de la limite une notion cartésienne dans son Descartes selon l'ordre des raisons, le tirant vers Leibniz d'une manière illégitime ${ }^{37}$. Partant de là, le raisonnement est simple : l'idée de limite n'est pas elle-même claire et distincte. Or la Règle VIII nous apprend que: "Si dans la série des objets de recherche il s'en présente un dont notre entendement ne puisse acquérir une intuition satisfaisante, il faut s'arrêter là : on ne doit point examiner ceux qui suivent, mais se dispenser d'une peine superflue ${ }^{38}$." Donc, il se comprend aisément que Descartes ne puisse accepter tout ce qui suit du problème de la limite, c'est-àdire, d'une manière générale, la question de l'infinitésimal elle-même.

Aussi est-il absurde d'écrire, comme le fait J. D. North, que le concept cartésien de limite non seulement est à l'origine de celui de l'infini mais encore influencera Newton, dont il est dit: "In playing with words, making limit a negative concept and therefore infinite a positive one, he is following in Descartes' footsteps ${ }^{39}$. " Il y a ici au moins trois erreurs. Premièrement, donner au concept de limite une place fondamentale chez Descartes. Deuxièmement, déduire ( «therefore ») la positivité de l'infini de la négativité de la limite alors même que Descartes ne cesse de répéter la priorité d'un infini

35. Belaval, 1960, p. 301.

36. Vuillemin, 1960, p. 95.

37. Belaval, 1955, p. 442-443 : "On pourrait contester l'authenticité cartésienne de certaines notions. Nous ne sommes pas sûr que, parlant de limite (I, 61, 220-221, etc.), M. Gueroult n'introduise en son commentaire une notion leibnizienne. "

38. Regulae VIII; AT X, 392. Cette règle constitue aussi une justification à ce qu'il ne faille point tenter de comprendre l'infini.

39. North, 1983, p. 122. 
dont la positivité ne signifie d'ailleurs rien d'autre (de telle sorte que « déduire la positivité de... » est une contradiction dans les termes, puisque la positivité est, par définition, première). Troisièmement, déclarer Newton cartésien pour avoir repris cette structure qui n'est pas chez Descartes!

En conclusion, si Descartes semblait avoir à sa portée une bonne partie des outils nécessaires à la génération du calcul infinitésimal, il n'en fit rien. Paralysé par le manque de rigueur de cette fiction seulement utile, il refuse de «négliger comme nulles ${ }^{40}$ — selon l'expression de Pascal qui, lui, l'acceptera sans peine - les quantités infiniment petites. C'est la méthode cartésienne qui dédaigne ici le peu de rigueur du "négliger" de l'évanouissement et demande à la démonstration mathématique d'être un continu — ou continûment légitimée - sans jamais laisser se faire, ne serait-ce qu'un instant, un saut illégitime, fûtil pragmatique. Il applique, en quelque sorte, sa rhétorique de l'attention, qui est une rhétorique contre la relâche ${ }^{41}$, au domaine des mathématiques ${ }^{42}$.

\section{B. Réfutation d'un soi-disant cas d'utilisation du passage à la limite en métaphysique}

Vuillemin remarque qu'un certain texte des Réponses aux Quatrièmes Objections semble utiliser le passage à la limite ${ }^{43}$, alors que celui-ci est refusé aux mathématiques. Si, comme nous le défendons, la fuite de Descartes est liée à des impératifs métaphysiques, pourquoi donc, au sein même de sa métaphysique, y aurait-il précisément ce avec quoi elle est prétendument incompatible? Il nous faut examiner l'argument de Vuillemin de plus près.

Dans les Quatrièmes Objections, Arnauld refuse la preuve cartésienne par les effets : le principe de causalité ne s'applique qu'aux choses finies (il faut

40. « On n'augmente pas une grandeur continue lorsqu'on lui ajoute, en tel nombre que l'on voudra, des grandeurs d'un ordre d'infinitude inférieur. Ainsi les points n'ajoutent rien aux lignes, les lignes aux surfaces, les surfaces aux solides; ou, pour parler en nombres - comme il convient dans un Traité d'Arithmétique - , les racines ne comptent pas par rapport aux carrés, les carrés par rapport aux cubes et les cubes par rapport aux carro-carrés. En sorte qu'on doit négliger comme nulles, les quantités d'ordre inférieur» (Pascal, 1908-1914, t. III, p. 367). Voir aussi: «J'estime qu'il n'est pas légitime et conforme à la géométrie d'admettre la méthode de démontrer par les indivisibles ou (comme j'ai coutume de les nommer) par les hétérogènes, que le célèbre géomètre Bonaventure Cavalieri a mis en lumière. Cette méthode célèbre passe des lignes aux surfaces, des superficies aux solides, et une égalité ou proportion trouvée pour les lignes donne une conclusion appliquée aux superficies. Par cette manière de raisonner on n'aboutit absolument à rien de certain à moins qu'on ne la ramène à l'homogénéité » (cité par Russo, 1962, p. 308).

41. Sur la relâche, voir par exemple les lettres à Elisabeth, de mai ou juin 1645, AT IV, 220, et du 6 octobre 1645, AT IV, 307.

42. Parce que « je ne puis pas ouvrir les yeux des lecteurs, ni les forcer d'avoir de l'attention aux choses qu'il faut considérer pour connaître clairement la vérité [...] je ne puis pas donner de l'esprit aux hommes, ni faire voir ce qui est au fond d'un cabinet, à des gens qui ne veulent pas entrer dedans pour le regarder " (à Mersenne, du 21 janvier 1641; AT III, 283-285), l'auteur s'adresse à l'attentus lector, au "mentis purae et attentae» (Regulae, III, AT, X, 368). La notion de "Rhetoric of attention « domine le chapitre III de Carr, 1990. Voir aussi Rodis-Lewis, 1950, p. 182.

43. Vuillemin, 1960, note III, p. 144-146. 
que la cause et l'effet soient distincts : la notion de causa sui est donc contradictoire dans les termes).

Descartes répond ceci :

Certes, il me semble que M. Arnauld a fait en ceci la même chose que si (après Archimède, parlant des choses qu'il a démontrées de la sphère par analogie aux figures rectilignes inscrites dans la sphère même, aurait dit: si je pensais que la sphère ne pût être prise pour une figure rectiligne, ou quasi rectiligne, dont les côtés sont infinis, je n'attribuerais aucune force à cette démonstration, parce qu'elle n'est pas véritable, si vous considérez la sphère comme une figure curviligne, ainsi qu'elle est en effet, mais bien si vous la considérez comme une figure rectiligne dont le nombre de côtés est infini). Si, dis-je, M. Arnauld, ne trouvant pas bon qu'on appelât ainsi la sphère, et néanmoins désirant retenir la démonstration d'Archimède, disait : si je pensais que ce qui se conclut ici, se dût entendre d'une figure rectiligne dont les côtés sont infinis, je ne croirais point du tout cela de la sphère, parce que j'ai une connaissance certaine que la sphère n'est point une figure rectiligne. Par lesquelles paroles il est sans doute qu'il ne ferait pas la même chose qu'Archimède, mais qu'au contraire il se ferait un obstacle à soi-même et empêcherait les autres de bien comprendre sa démonstration ${ }^{44}$.

\section{Vuillemin commente :}

Descartes répond par un raisonnement qui utilise le passage à la limite. Comme Archimède démontre des propriétés pour le solide rectiligne inscrit dans la sphère et l'applique à la sphère même, conçue comme un solide ayant une infinité de faces, de même le philosophe est légitimé, si le principe de causalité peut être à bon droit appliqué aux choses et aux idées des choses finies, à l'appliquer à l'infini même et à l'idée que nous en recevons ${ }^{45}$.

Or nous contestons la pertinence d'une équivalence entre «le passage à la limite en mathématiques», lequel est quantitatif, et « le passage à la limite en métaphysique », qui, lui, est qualitatif : Descartes ne fait qu'utiliser un raisonnement qui n'a de "passage à la limite » que la forme. Il n'est donc pas ici question du problème de la limite tel qu'il se manifeste en mathématiques.

Par ailleurs, Vuillemin ajoute que Descartes semble utiliser un raisonnement qui apparaîtra explicitement chez Leibniz sous le nom de principe de continuité et se manifeste ainsi dans le texte leibnizien :

Lorsque la différence de deux cas peut être diminuée au-dessous de toute grandeur donnée, in datis, ou dans ce qui est posé, il faut qu'elle puisse se trouver aussi diminuée au-dessous de toute grandeur donnée in quaesitis, ou dans ce qui en résulte; ou, pour parler plus familièrement, lorsque les cas (ou ce qui est donné) s'approchent continuellement et se perdent enfin l'un dans l'autre, il faut que les suites, ou événements (ou ce qui est demandé) le fassent aussi ${ }^{46}$.

44. Réponses aux Quatrièmes Objections; AT IX-1 189.

45. Vuillemin, 1960, 145. Alquié, 1950, p. 225-226, parle lui aussi de "passage à la limite, ainsi opéré en ce qui concerne le principe de causalité».

46. Leibniz, Nouvelles de la République des Lettres, mai 1687, dans Leibniz, 1960, p. 744. 
Le principe de continuité leibnizien enveloppe ainsi un passage aux limites : Leibniz applique à sa métaphysique les procédés inventés pour le calcul infinitésimal. Mais il ne nous semble pas non plus adéquat d'attribuer ce principe de continuité à Descartes, sur le problème du fini et de l'infini, puisque la continuité entre ces deux notions est chez lui inacceptable, en ce qu'il n'y a pas entre elles une différence de degrée, mais de nature. Cela est confirmé par Belaval, qui infirme l'attribution de la notion de limite que fait Gueroult à Descartes, par le fait qu'une telle notion présuppose un principe de continuité que Descartes est loin de considérer : "Cette notion implique un principe de continuité que Descartes, non seulement n'a pas généralisé à tout le domaine mathématique (puisqu'il ne peut passer des courbes algébriques aux courbes transcendantes), mais encore qu'il n'a pas songé à étendre à sa Mécanique ${ }^{47}$.» Il faut conclure que Vuillemin, comme Gueroult, tire ici Descartes vers Leibniz.

En conclusion, il ne semble pas que ce prétendu cas d'utilisation de passage à la limite en Métaphysique soit inquiétant pour notre affaire.

\section{L'indéfini : existence et statut du «plus grand nombre »}

On sait donc comment Descartes a contourné la question de l'infinitésimal, comme celle de l'infini en mathématiques. Reste que, malgré tout, il ne peut éviter de la rencontrer, ici et là, dans un traité de géométrie comme au détour d'une correspondance métaphysique. Comment, dans ce cas, réagit-il ? Il ne répond à la question que par l'indétermination de l'in-défini. Et l'exemple parfait d'un tel comportement se trouve particulièrement manifeste dans le problème du nombre infini, ou du "plus grand nombre ». Nous verrons, tout d'abord, comment Descartes, par la finitude de l'entendement condition du nombre, écarte d'emblée la notion même de "nombre infini ", dans le but de mieux substituer à l'infinité de la formule classique, et ici refusée, le simple maximum d'un "plus grand nombre». Il s'agira ensuite de préciser l'in-définité de ce plus grand nombre, pour ne pas pouvoir prouver son existence : car c'est effectivement de cette position concernant son existence que découlera son statut, à savoir celui de nombre indéfini.

A. Comment la finitude de l'entendement infirme la notion de «nombre infini »

À la base des mathématiques se trouve le nombre. À la base de la question de l'infini en mathématiques, donc, se trouve celle du nombre infini. Or, le nombre relève de l'entendement. Si donc il peut seulement ensuite être dit luimême au fondement des mathématiques, c'est que, par continuité, les mathématiques, à travers le nombre, ont pour condition l'entendement. Or, et c'est de là que vient le problème lorsque se pose la question de l'infini en mathématiques, l'entendement est fini : «ma nature [...] est finie et bornée ${ }^{48}$ »

47. Belaval, 1955, p. 443.

48. Méditation III; AT IX-1 37. 
La conclusion ne saurait tarder: n'ont de sens ni un «nombre infini» ni toute investigation «infinitisante» en mathématiques.

C'est donc bien la finitude de l'entendement qui est le nerf du rejet cartésien de l'infini mathématique, comme le confirmera Cantor dans une critique acerbe :

Les mots «entendement fini » que l'on entend si souvent n'offrent, je le pense, aucun caractère pertinent : si bornée que soit en vérité la nature humaine, elle porte pourtant en elle, c'est inhérent, une très grande part d'infini, et j'irais jusqu'à dire que si elle n'était pas elle-même infinie sous bien des rapports, on ne saurait expliquer la ferme assurance et la pleine certitude où nous nous savons tous unis, s'agissant de l'être de l'absolu' ${ }^{49}$.

Le fait que Cantor, pour asseoir sa position infinitiste, soit amené à s'en prendre à la finitude de l'entendement cartésien non seulement confirme, d'une part, que, d'une manière générale, l'entendement est condition du nombre et, d'autre part, que, chez Descartes, la finitude de l'entendement est condition du rejet de l'infinité du nombre, mais aussi explique pourquoi Cantor préférera Spinoza, chez qui l'entendement est infini - puisqu'il comprend $d^{50}$ l'infini - pour jeter les bases philosophiques de son infinitisme mathématique tel qu'il est à l'œuvre dans sa théorie des ensembles ${ }^{51}$.

\section{B. Qu'on ne peut prouver l'existence du «plus grand nombre »: indéfinité}

Descartes rejette donc d'emblée la notion de «nombre infini ». Il n'en faudra pas moins à Leibniz, qui sur ce point partage les vues cartésiennes, pour en déduire sa simple finitude : si le nombre n'est pas infini, c'est qu'il est fini. C'est effectivement en niant l'existence de l'infini catégorématique, infini dénombrable ( «Il n'est pas donné d'Infini catégorématique, c'est-à-dire ayant en acte des parties infinies formellement $\left.{ }^{52} »\right)$, que Leibniz en vient à nier la notion de nombre infini : «Il n'y a point de nombre infini, ni de ligne ou autre quantité infinie, si on les prend pour de véritables touts [...] Les écoles ont voulu dire cela en admettant un infini syncatégorématique, comme elles parlent, et non pas

49. Cantor, 1932, p. 176-177.

50. À prendre dans les deux sens : si précisément l'entendement comprend (comprehendere) l'infini, c'est pour le comprendre (inclusion, qui serait identité puisqu'on ne peut enfermer l'infini : l'entendement est lui-même infini).

51. On notera cependant que l'infinitude de l'entendement n'empêche pas Spinoza d'établir l'impossibilité d'un nombre infini, puisque "l'infini et le nombre s'excluent » (Gueroult, 1966, p. 401): «Ni le nombre, ni la mesure, ni le temps, puisqu'ils ne sont que des auxiliaires de l'imagination, ne peuvent être infinis, car autrement le nombre ne serait plus nombre, ni la mesure, mesure, ni le temps, temps; de là on voit clairement pourquoi, confondant ces trois êtres de raison avec les choses réelles dont on ignore la vraie nature, on nie fréquemment l'infini en acte » (Lettre XII à Louis Meyer, dans Spinoza, 1954, p. 1097). Si l'infini et le nombre s'excluent, c'est que, pour le coup, comme chez Descartes, seul l'entendement peut concevoir l'infini, alors que le nombre est généré par l'imagination qui, elle, ne peut s'imaginer l'infini.

52. À Des Bosses, le $1^{\text {er }}$ septembre 1706, dans Leibniz, 1960, t. II, p. 314. 
l'infini catégorématique ${ }^{53}$. Et, de cette négation, il déduit l'affirmation de sa finitude : «Il appartient à l'essence du nombre, de la ligne, et d'un tout quelconque, d'être borné f $^{54}$.» La position de Descartes est plus complexe pour au moins deux raisons.

Premièrement, son rejet de la notion de "nombre infini " n'implique pas le rejet de son existence, qu'il ne trouve pas a priori absurde. À Burman qui demande: "Mais il y aurait dans l'hypothèse envisagée un nombre infini ?", il aurait répondu : "Et où serait l'absurdité ? » ${ }^{55}$. Hannequin s'empresse d'en déduire que le plus grand nombre cartésien, pour cette raison et le fait que, chez Descartes, un infini puisse être plus grand qu'un autre, n'annonce rien de moins que le transfini cantorien:

Ainsi Descartes ne trouve absurde a priori ni l'existence d'un nombre infini [...] ou même celle de plusieurs nombres infinis différents les uns des autres ni la possibilité de concevoir entre eux des rapports qui ne répondent point nécessairement aux rapports des nombres finis; et nous nous contentons de dire qu'en cela il ne faisait qu'énoncer des propositions rendues plus que plausibles par de récentes spéculations sur l'infini proprement dit ou sur le transfini ${ }^{56}$ [voir notamment les travaux de M. George Cantor] .

C'est que, de la même manière que l'existence d'un nombre infini ne serait pas absurde, il n'est pas absurde qu'un infini soit plus grand qu'un autre: on pourrait par exemple penser que la série «infinie » des nombres n'a pas de sens, que nous la posons comme telle, sans même savoir ce qu'elle signifie, puisque la série des nombres entiers et celle des nombres pairs constituent deux infinis dont l'un est le double de l'autre - Cantor, avant Russell, a justement montré qu'il y avait bijection entre ces deux ensembles - ce qui semble absurde, pour beaucoup, dont Spinoza lui-même: "Si chaque partie est infinie, il y a donc un infini deux fois plus grand qu'un autre infini, ce qui est également absurde $^{57}$. " Mais, pour Descartes, au contraire, il n'y a rien d'absurde à ce qu'un infini soit plus grand qu'un autre:

Mais, à propos de l'infini, vous m'en proposiez une question en votre lettre du 14 mars, qui est tout ce que j'y trouve de plus qu'en la dernière. Vous disiez que, s'il y avait une ligne infinie, elle aurait un nombre infini de pieds et de toises, et par conséquent que le nombre infini des pieds serait six fois plus grand que le nombre des toises ${ }^{58}$. - Concedo totum [ «j'accorde tout cela»]. - Donc ce dernier n'est pas infini. — Nego consequentiam [ "Je nie la conséquence»]. Mais un infini ne peut être plus grand que l'autre. - Pourquoi non? Quid

53. Leibniz, 1990, p. 124.

54. À Des Bosses le 11 mars 1706, dans Leibniz, 1960, t. II, p. 304.

55. Entretien avec Burman; AT V, 155 ; Descartes, 1981, p. 52.

56. Hannequin, 1896, p. 447, note.

57. Ethique, prop. 15, scolie, in Spinoza, 1954, 380. Le scolie de la prop. 15 discute de manière substantielle de divers problèmes touchant l'infini, dans le but d'établir l'infini de l'étendue, en tant qu'elle exprime un Dieu infini.

58. 1 toise $=6$ pieds. 
absurdi? [ QQu'y a-t-il d'absurde en cela ? »]. Principalement s'il est seulement plus grand in ratione finita, ut hic ubi multiplicatio per 6 est ratio finita, quoe nibil attinet as infinitum ["Selon un rapport fini, comme ici où la multiplication par 6 est un rapport fini qui ne touche en rien à l'infini »]. Et de plus, quelle raison avons-nous de juger si un infini peut être plus grand que l'autre, ou non? $\mathrm{Vu}$ qu'il cesserait d'être infini, si nous le pouvions comprendre ${ }^{59}$.

On ne peut identifier un nombre infini comme absolu avec un nombre « infini » qui serait relatif, puisque six fois plus grand qu'un autre. De là, on tirera deux remarques.

D’une part, cet argument logique trouve un fondement dans la méthode cartésienne des moyennes proportionnelles décrite par la Règle VI des Regulae: " Je remarque en effet, pour commencer, qu'il n'a pas été plus difficile de trouver le double de 6 que le double de 3 ; et que pareillement, en toutes choses, une fois trouvée la proportion qui existe entre deux grandeurs quelconques, on peut trouver d'autres grandeurs en nombre indéfini, qui ont entre elles la même proportion $^{60}$. " En découvrant les moyennes proportionnelles, Descartes fait du nombre une grandeur numérique toujours relative, en tant qu'elle est toujours à la fois le produit d'un nombre et la moyenne proportionnelle d'un autre. De cette manière, le nombre ne peut être "infini» et incompréhensible, puisqu'il est relatif et exprimable ${ }^{61}$.

Et, d'autre part, Yvon Belaval exploite cet argument pour mettre en parallèle, chez Descartes, le couple infini/indéfini et celui des ensembles infinis dénombrables/ensembles transfinis :

Descartes semble même pressentir la différence que les mathématiciens ont, depuis, institué entre ensembles infinis dénombrables et ensembles transfinis : des premiers, il donne un exemple lorsqu'il affirme ne rien voir d'absurde à multiplier par 6 un nombre infini de toises, ni même dans la multiplication infinie de l'indéfini qui est égale à l'infini; et avec ce que l'entendement divin conçoit audelà du plus grand nombre (majus numero), ne pénétrons-nous pas dans le transfini?

Et, comme pour répondre à Hannequin, et contester qu'il s'agit là effectivement de transfini, Belaval poursuit : «Mais il aurait fallu que Descartes passât du nombre cardinal à la considération de l'ordinal, pour concevoir le transfini ${ }^{62} »-$ ce qui d'ailleurs est inexact.

Deuxièmement, la non-absurdité de l'existence d'un «nombre infini » n'implique pas l'absurdité de son inexistence. Car toute l'originalité de l'indéfini cartésien - qui se révèlera effectivement occuper le problème du « plus grand nombre» — réside précisément en cette possibilité de contourner la logique

59. À Mersenne le 15 avril 1630; AT I, 146-147.

60. Regulae VI; AT X, 385.

61. Sur le problème des moyennes proportionnelles, on peut voir particulièrement l'analyse de Costabel, 1982.

62. Belaval, 1960, p. 226. 
binaire qui fait du fini et de l'infini des contradictoires dont la négation de l'un, en l'occurrence du second, impliquerait l'affirmation de l'autre, en l'occurrence du premier. Descartes, de la négation de l'infinité du nombre, ne déduit pas sa finitude, puisqu'il ajoute qu'il ne refuse pas moins l'inexistence que l'existence à ce prétendu "nombre infini »: face à l'idée d'un "nombre infini ", Descartes, d'une part, comme on le sait, refuse donc d'en accepter l'existence, puisqu'un tel nombre relève de l'entendement fini et, d'autre part, refuse effectivement avec tout autant de force de la nier en le déclarant contradictoire, ce qui était pourtant relativement courant à l'époque, chez les scolastiques, Leibniz lui-même, ou Mersenne ${ }^{63}$, à qui Descartes répond ceci : «De cela seul que j'aperçois que je ne puis jamais, en nombrant, arriver au plus grand de tous les nombres, et que de là je connais qu'il y a quelque chose en matière de nombrer qui surpasse mes forces, je puis conclure nécessairement, non pas à la vérité qu'un nombre infini existe, ni aussi que son existence implique contradiction, comme vous dites ${ }^{64}$. "Si donc j'ai le pouvoir de nombrer à l'infiniet cette infinie puissance de nombrer n'est autre qu'une manifestation de l'infini de ma volonté - je n'ai jamais pour autant nombré le plus grand nombre. Par conséquent, je ne peux rien déduire de son existence. L'indécidabilité de l'existence du plus grand nombre - puisque ni la proposition de l'existence d'un «nombre infini » ni la proposition contraire ne sont démontrables implique, entre autres, les deux remarques suivantes.

D'abord, le fait que je ne puisse déduire ni l'existence ni la contradiction $\mathrm{du}$ «nombre infini » implique que le principe du tiers exclu devienne ici inapplicable. Pour cette raison, Yvon Belaval parle de l'«intuitionnisme» mathématique de Descartes, qu'il dit comparable aujourd'hui à celui de Brouwer, Weyl, Lebesgue ou Borel, dans la mesure où, comme chez eux, le principe du tiers exclu cesse ici d'être valable dans un ensemble infini ${ }^{65}$. Remarquons par ailleurs que Descartes n'accorde de toute façon que peu d'importance à ce principe:

Au premier sens, on peut dire que impossibile est idem simul esse et non esse est un principe, et qu'il peut connaître l'existence d'une chose, mais seulement à faire que, lorsqu'on la connaît, on en confirme la vérité par un tel raisonnement. Il est impossible que ce qui est ne soit pas; or je connais que telle chose est; donc je connais qu'il est impossible qu'elle ne soit pas. Ce qui est de bien peu d'importance, et ne nous rend de rien plus savants ${ }^{66}$.

Et si le principe du tiers exclu est « de bien peu d'importance », c'est que Dieu est au-dessus du principe de non-contradiction qui le conditionne: "Dieu ne peut avoir été déterminé à faire qu'il fût vrai, que les contradictoires ne peuvent être ensemble, et que, par conséquent, il a pu faire le contraire ${ }^{67}$. »

63. Les Secondes Objections, AT VII, 124, disaient : "est numerus ille impossibilis ".

64. Réponses aux secondes objections; AT IX-1, 109-110, spn.

65. Belaval, 1960, p. 221. Ce qui est inexact dans le cas de Borel, qui acceptait l'infini dénombrable (.

66. À Clerselier de juin ou juillet 1646; AT IV, 444.

67. À Mesland du 2 mai 1644; AT IV, 118. 
Ensuite, on notera que Leibniz, dans ses Animadversiones ${ }^{68}$, profitera de cette indétermination pour dénoncer une soi-disant contradiction: Descartes, d'une part, refuse de nier ou d'affirmer l'existence du dernier terme dans une série divergente (à juste titre, par définition, car une série divergente n'a pas de limite $)^{69}$ et, d'autre part, affirme la divisibilité de la matière à l'infini; de telle sorte qu'il y a ensemble un nombre infini indécidable et un nombre infini de parties décidable. On pourrait répondre à Leibniz en utilisant les textes de Descartes lui-même, et contester qu'il y a effectivement contradiction en distinguant divisibilité et division: "Pensez que chaque corps peut être divisé en des parties extrêmement petites. Je ne veux point déterminer si leur nombre est infini ou non; mais du moins il est certain qu'à l'égard de notre connaissance il est indéfini, et que nous pouvons supposer qu'il y en a plusieurs millions dans le moindre petit grain de sable qui puisse être aperçu de nos yeux ${ }^{70}{ }$; ou encore: "De ce que je ne puis comprendre [comprehendere] une infinité de divisions en une quantité finie, il ne s'ensuit pas que l'on puisse venir à une dernière, après laquelle cette quantité ne puisse plus être divisée; mais bien il suit seulement que mon entendement, qui est fini, ne peut comprendre [capere] l'infini ${ }^{71}$. " Dans ces textes est manifeste la distinction entre la possibilité d'être divisé (divisibilité) et la division effective. La contradiction soulevée par Leibniz n'a plus lieu d'être si l'on répartit l'indécidabilité du nombre infini dans la division et la décidabilité d'un nombre infini de parties dans la seule divisibilité, qui est aussi peu concurrente de la première que la puissance de l'acte.

C'est ainsi que l'indécidabilité de l'existence du «nombre infini » lui donne un statut d'indéfini - ce dont on ne peut prouver les bornes - puisqu'on ne peut le prouver infini : "Et il suppose aussi qu'il ne peut y avoir de nombre infini, etc., ce qu'il ne saurait prouver non plus ${ }^{72}$. Pour cette raison, et de la même manière que "parce que nous ne saurions imaginer tant d'étoiles que Dieu n'en puisse créer davantage, nous supposerons que leur nombre est indéfini, et ainsi du reste ${ }^{73}$ ", le "plus grand nombre » reste indéfini. L'Entretien confirmera cette conclusion - tout en manifestant, une fois de plus, la dépendance des mathématiques à l'égard de la physique - en ajoutant, comme une preuve supplémentaire: "Ce nombre infini ne se rencontre-t-il pas dans la division de la quantité, etc. ? ${ }^{74}$ ", alors que la divisibilité de la matière en question est indéfinie et non infinie.

68. Animadversiones de Paris, art. 26-27.

69. Le passage du nombre infini à la suite — ou série — infinie se fait naturellement, puisque la question de l'infini, en mathématiques, peut elle-même être réduite au fait que, de chaque nombre, et aussi grand qu'il soit, on pourra toujours trouver un nombre plus grand encore, ne serait-ce qu'en lui ajoutant 1 , et ainsi indéfiniment.

70. Le Monde XI; AT XI, 12.

71. Réponses aux Premières Objections; AT IX-1, 85.

72. À Mersenne le 28 janvier 1641; AT III, 294, spn.

73. Principes I, 26; AT IX-2, 36.

74. Entretien avec Burman; AT V, 155 ; Descartes, 1981, p. 52. 


\section{Conclusion : les raisons de la prudence cartésienne}

La question de l'infinitésimal exprime de manière paradigmatique la prudence de Descartes face au problème de l'infini en mathématiques. Conclure sur ce point implique maintenant d'explorer les raisons supposées de l'esquive cartésienne, afin de comprendre pourquoi Descartes s'est tant évertué à contourner soigneusement toute manifestation de l'infini en mathématiques : quel est donc le moteur de la prudence cartésienne?

Certains voudraient y voir des raisons internes aux mathématiques ou à la physique, dans le seul but de trouver chez Descartes une autorité de plus à la légitimation de l'indépendance du scientifique par rapport au philosophique, voire même du logique par rapport au métaphysique. Mais dans le cadre de l'histoire de la philosophie, laquelle commande de ne pas interpréter l'auteur au-delà de ce qu'on y peut lire, et particulièrement dans le cas de Descartes, il est absolument incontestable que l'indéfini mathématique présuppose l'indéfini physique, lequel présuppose à son tour l'infini divin, et que, de cette manière, on ne peut raisonnablement y comprendre une quelconque indépendance qu'en la posant soi-même de sa lecture. Nous limitant, pour notre part, aux textes cartésiens, il est aisé de montrer, de trois manières, comment la fuite de Descartes face à la question de l'infini en mathématiques est liée à des impératifs métaphysiques, comme tous les grands commentateurs de Descartes le reconnaissent sans peine.

D'abord, on peut réaliser la dépendance de l'indéfini mathématique envers l'infini divin, par le seul choix du vocabulaire qui nous contraint à ne parler de l'infini mathématique qu'en terme d'indéfini, parce que Dieu seul, qui est passé avant, pouvait ravir l'infini. L'indéfini étant ainsi nommé par défaut, pour désigner tout ce qui nous semble infini sans être Dieu, donc sans l'être positivement, l'indéfini mathématique souffre d'une dette envers l'infini divin au moins linguistique.

Ensuite, lorsqu'Yvon Belaval écrit: "On remarque quelles idées se retrouvent toujours dans l'esprit de Descartes lorsqu'il aborde le problème du plus grand nombre : l'idée de Dieu, celle d'une puissance de nombrer, celle du nombre lui-même et celle, enfin, de l'existence de ce nombre ${ }^{75}$ ", il faut comprendre l'ordre de l'énumération comme n'étant pas hasardeux. L'idée de Dieu est en effet d'abord là, toujours antécédente, comme l'exige l'ordre des raisons dans les Méditations: c'est uniquement parce que j'ai en moi l'idée de Dieu et que de cette manière je peux démontrer son existence, que je pourrai faire de Dieu un principe épistémologique dont la véracité permettra, dans une dialectique descendante (application), de refonder la possibilité même de la science, dont le nombre fait partie. C'est ainsi que « cette puissance de nombrer confirme la preuve de Dieu. C'est par elle que je puis produire les nombres, mais cette cause immédiate du nombre est elle-même l'effet d'une cause supérieure, car je ne suis pas cause de moi, ni cause de ma conservation ${ }^{76}{ }^{\prime}$. De

75. Belaval, 1960, p. 222.

76. Belaval, 1960, p. 223-224. Voir les Réponses aux Secondes Objections, AT VII, 139. 
ce point de vue, parce que l'existence de Dieu est condition de mon idée du nombre, l'infini divin est condition de l'indéfini mathématique.

Enfin, cela est aussi aisément démontrable par l'indéfini physique, en utilisant la transitivité du syllogisme suivant: On sait que l'indéfini physique dépend de l'infini divin, or l'indéfini mathématique dépend de l'indéfini physique, donc l'indéfini mathématique dépend de l'infini divin.

Que l'indéfini physique dépende de l'infini divin, nous l'avons montré ailleurs, et notamment à travers l'utilisation de Dieu dans les preuves de l'indéfinité de l'univers ${ }^{77}$. C'est pourquoi Françoise Monnoyeur n'hésite pas à déclarer : "La considération sur l'infinité du monde est envisagée par rapport à l'infinité divine et se trouve ainsi en étroite dépendance de celle-ci ${ }^{78}$. En accord avec Michel Fichant ${ }^{79}$, toute interprétation positiviste de la physique cartésienne, qui à vrai dire ne se fait plus guère depuis Louis Liard, qui la caractériserait par «l'absence de toute idée métaphysique » ${ }^{80}$, doit être abandonnée, si l'on veut être fidèle aux textes cartésiens. Le métaphysique, dans l'œuvre de Descartes, ne peut en être détachée sans blessures ${ }^{81}$.

Que l'indéfini mathématique dépende ensuite de l'indéfini physique, c'est la définition même du célèbre géométrisme cartésien, qui fait poursuivre Belaval: «Chez Descartes, tout l'infini quantitatif, dont traitent les mathématiques, est réductible à l'étendue géométrique ${ }^{82}$. On peut à cet égard remarquer que si Descartes a adopté la recta linea comme type de la quantité, c'est précisément pour ne pas connaître le nombre irrationnel en tant que nombre. Celui-ci, selon la définition de Cauchy, étant la limite des diverses fractions qui en fournissent des valeurs de plus en plus approchées, on comprend que Descartes n'ait pu, contrairement à Leibniz, participer à sa génération, d'une part pour le problème que lui pose la notion de limite, laquelle inclut le nombre irrationnel à la question de l'infini mathématique qu'il ne veut point résoudre, et d'autre part parce qu'il aurait fallu, pour cela, substituer au quantitatif cardinal le qualitatif ordinal. Et cette même étendue géométrique présuppose la matière et le mouvement de la physique : "Chez Descartes, cet infini - ou cet indéfini - mathématique, Dieu l'explique seulement en tant que cause : cet infini nous est donné dans l'essence de la matière et du mouvement, encore "plus aisé à concevoir que la ligne des géomètres " ${ }^{83}$, parce qu'il engendre les lignes ${ }^{84}$.» On comprend ainsi que «Descartes ne posait le problème du plus grand nombre que par rapport au monde ${ }^{85} »$.

77. Voir notre article «Descartes : indéfinité de et dans l'univers ", à paraître.

78. Monnoyeur, 1992, p. 85.

79. Fichant, 1998, p. 65.

80. Liard, 1882, p. 66.

81. Voir notamment Gilson, 1930, p. 176.

82. Belaval, 1960 , p. 223-224.

83. Le Monde, VII; AT XI, 40.

84. Belaval, 1960, p. 223-224.

85. Belaval, 1960, p. 272. 


\section{Philosophiques / Automne 2007}

On peut donc légitimement conclure que, par extension, l'indéfini mathématique dépend de l'infini divin: "l'infini mathématique présuppose un infini de nature spirituelle ${ }^{86} »$. Vuillemin confirme: «Descartes peut donc bien être regardé comme l'un des fondateurs du Calcul intégral, techniquement parlant. Mais l'interdit métaphysique qu'il faisait peser sur la classe des transcendantes lui interdisait de donner droit de cité à sa découverte ${ }^{87}$.»

Ainsi, Descartes a développé sa pensée scientifique dans un espace soumis à ne pas pouvoir dépasser la voûte métaphysique; et c'est précisément par cette limitation qu'en partie «s'explique la fuite de Descartes devant toute spéculation mathématique sur l'infini, bien que son génie eût commencé à en explorer les voies ${ }^{88} »$. Cela ne suffit pas, pourtant. La prudence épistémologique de Descartes face à la question de l'infini, partagée d'ailleurs par nombre de penseurs de Copernic à Kant, est aussi et surtout la manifestation d'un esprit attaché à ne pas transgresser l'ordre des raisons ${ }^{89}$. Le moteur de sa prudence reste une contrainte, mais elle est double: voûte métaphysique et rigueur méthodologique.

\section{Bibliographie}

\section{Descartes}

Nous citons Descartes dans l'édition de référence d'Adam et Tannery en 12 volumes, Paris, Vrin, 1897-1913, dans sa nouvelle présentation par P. Costabel et B. Rochot, Paris, Vrin-CNRS, 1966. L'Entretien avec Burman est publié dans une édition distincte: Descartes, Entretien avec Burman, édition de Jean-Marie Beyssade, Paris, PUF, 1981.

\section{Littérature secondaire}

Alquié, Ferdinand. La découverte métaphysique de l'homme chez Descartes, Paris, PUF, 1950.

—. Édition et notes de Descartes, Euvres Philosophiques, t. II, Paris, Bordas, 1992.

Banfi, Antonio. "Galilée, Descartes et Vico », in Descartes, Cahiers de Royaumont, Philosophie n. II, Paris, Editions de Minuit, 1957, p. 376-392.

Belaval, Yvon. "Descartes selon l'ordre des raisons, d'après M. Gueroult ", Revue de métaphysique et de morale, 1955, p. 442-443.

—. Leibniz critique de Descartes, Paris, Gallimard, 1960.

Berkeley, George. CEuvres, vol. I, édition de G. Brykman, Paris, PUF, 1985.

86. Belaval, 1960, p. 224.

87. Vuillemin, 1960, p. 73, souligné par nous (spn). Plus généralement, «Leibniz d'une part, Newton et Kant de l'autre fonderont leurs systèmes sur les ruines du cartésianisme en accordant la première place, celui-là à l'idée de virtualité, ceux-ci aux constructions mécaniques, que Descartes, mathématicien, avait naturellement rencontrées pour résoudre des problèmes techniques, mais que la cohérence de sa méthode métaphysique l'avait contraint de ne traiter qu'aux marges des découvertes qu'il jugeait essentielles à sa pensée. »

88. Gueroult, 1968, p. 521.

89. Sur Copernic notamment, voir Seidengart, 2006. Je remercie l'évaluateur anonyme de Philosophiques d'avoir attiré mon attention sur ce point. 
Brunschvicg, Léon. "La révolution cartésienne et la notion spinoziste de la Substance ", Revue de métaphysique et de morale, 1904, p. 755-798.

—. "Mathématique et métaphysique chez Descartes ", Revue de métaphysique et de morale, 1927. p. 277-324.

Cantor, Georg. Gesammelte Abhandlungen Mathematischen und Philosophischen Inhalts, Berlin, J. Springer, 1932.

Carnot, Lazare. Réflexions sur la métaphysique du calcul infinitésimal, 2 vol., Paris, Duprat, 1797.

Carr, Thomas A. Descartes and the Resilience of Rhetoric, Carbondale, Southern Illinois University Press, 1990.

Cléro, Jean-Pierre. "La méthode des fluxions de Newton", dans Divers aspects de l'Infini en mathématiques et en philosophie, Cahiers pédagogiques de philosophie et d'histoire des mathématiques, ministère de l'Éducation nationale, académie de Rouen, 1989-90, fasc. 2, p. 169-190.

Costabel, Pierre. «La solution par Descartes du problème des moyennes proportionnelles (Règle VI) ", dans Démarches originales de Descartes savant, Paris, Vrin, 1982, p. 49-62.

Couturat, Louis. De l'infini mathématique, Paris, Alcan, 1896.

Enriques, Federico. "Descartes et Galilée », Revue de métaphysique et de morale, 1937, p. 221-235.

Fénélon, François de Salignac de la Motte. Démonstration de l'existence de Dieu, tirée de la connaissance de la Nature, et proportionnée à la faible intelligence des plus simples, Paris, Jacques Estienne, 1712.

Fichant, Michel. Science et métaphysique dans Descartes et Leibniz, Paris, PUF, 1998.

Galilée, Galilei Galileo. Discours et démonstrations mathématiques concernant deux sciences nouvelles, traduit par Maurice Clavelin, Paris, A. Colin, 1970.

Gillispie, Charles Coulston, et Adolf Pavlovich Iushkevich. Lazare Carnot savant et sa contribution à la théorie de l'infini en mathématiques, Paris, Vrin, 1979.

Gilson, Étienne. Études sur l'histoire de la formation du système cartésien, Paris, Vrin, 1930.

Gueroult, Martial. "La lettre de Spinoza sur l'infini », Revue de métaphysique et de morale, 1966.

$1 /{ }_{\mathrm{M}}^{3}$. Spinoza: Dieu, Ethique I, Paris, Aubier-Montaigne, 1968.

Houzel, Christian. "Descartes et les courbes transcendantes", dans J. Biard \& R. Rashed, dir., Descartes et le Moyen Âge, Paris, Vrin, 1997.

Gandt, François de. "Newton: la justification des infiniment petits et l'intuition du mouvement », dans F. Monnoyeur, dir., Infini des mathématiciens, infini des philosophes, Paris, Berlin, 1992, p. 147-157.

Hannequin, Arthur. "La preuve ontologique cartésienne défendue contre la critique de Leibniz », Revue de métaphysique et de morale, 1896, p. 433-458.

Leibniz, Gerhardt Wilhelm. Die Philosophischen Schriften von Gottfried Wilhelm Leibniz, édition de C. D. Gerhardt en 7 vol., Berlin, 1875, réed. Georg Olms Hildeshein, 1960.

—. Nouveaux Essais sur l'entendement humain, édition de Jacques Brunschwig, Paris, GF, Flammarion, 1990.

Liard, Louis. Descartes, Paris, G. Bailliere et C $C^{\mathrm{ie}}, 1882$.

Mahoney, Michael Sean. The Mathematical Career of Pierre de Fermat, Princeton, Princeton University Press, 1973. 
Marion, Jean-Luc. Sur la théologie blanche de Descartes, Paris, PUF, 1981.

Monnoyeur, Françoise. "L'infini et l'indéfini dans la théorie cartésienne de la connaissance ", dans F. Monnoyeur, dir., Infini des mathématiciens, infini des philosophes, Paris, Berlin, 1992.

North, John David. "Finite and Otherwise : Aristotle and some Seventeenth Century Views ", dans William R. Shea, dir., Nature Mathematized, Dordrecht, Reidel, 1983.

Pascal, Euvres de Pascal, édition de Léon Brunschvicg, Pierre Boutroux et Félix Gazie, 11 vol., Paris, Hachette, 1908-1914.

Rodis-Lewis, Geneviève. L'individualité selon Descartes, Paris, Vrin, 1950.

Russo, François. "Pascal et l'analyse infinitésimale ", Revue d'histoire des sciences, vol. 15, 1962, p. 303-320.

Seidengart, Jean. Dieu, l'univers et la sphère infinie: penser l'infinité cosmique à l'aube de la science classique, Paris, Albin Michel.

Sergescu, Petre. "L'infiniment petit mathématique du Moyen Âge au XIX ${ }^{\mathrm{e}}$ siècle », dans L'infini et le réel, Centre international de synthèse, Dix-huitième semaine de synthèse, Exposés et discussions, Paris, Albin Michel, 1955.

Shea, William R. "Descartes as critic of Galileo ", dans R. E. Butts \& J. C. Pitts, dir., New Perspectives on Galileo, Dordrecht, Reidel, 1978, p. 139-159.

Spinoza. Oeuvres Complètes, édition de Roland Caillois, Madeleine Francès et Robert Misrahi, Paris, Gallimard, 1954.

Vuillemin, Jules. Mathématiques et métaphysique chez Descartes, Paris, PUF, 1960. 